\title{
Reflection of Elastic Waves from Cylindrical Surfaces*
}

\section{S. AHLUWALIA, J. B. KELLER $\&$ E. RESENDE}

\author{
Communicated by J. B. KeLLER
}

0. Introduction. We shall consider the propagation of elastic waves and their reflection from a rigid cylinder having an arbitrary smooth curve as its cross section, according to the linear theory of elasticity. The propagation of a large class of waves can be described by means of the "progressing wave" expansion, which is explained in Section 1. The special case of time-independent progressing elastic waves was previously considered by Karal and Keller [1]. In Section 2 we show how to find the two reflected progressing waves, one a shear wave and the other a compressional wave, which result when an incident progressing wave hits a rigid cylinder. In Section 3 we calculate the reflected waves in detail for the special but important case of time independent progressing waves, which includes time harmonic waves. Although the method we use could be applied to inhomogeneous media, we shall treat only the homogeneous case.

The theory of progressing waves involves geometrical optics concepts such as rays, phase functions, amplitude functions, transport equations, laws of reflection, etc. Thus it is an asymptotic theory, valid for the short wavelength or high frequency components of a wave motion. When applied to transient waves or pulses, it is valid for the rapidly varying portions of them, such as the first motions. The theory of scalar progressing waves is given in detail by Lewis [2]. The diffraction of scalar progressing waves by the edge of a thin screen was considered by Ahluwalia and Lewis [3]. The reflection of scalar time-harmonic waves from a cylinder was treated by Keller, Lewis and Seckler [4], and their results apply also to time independent progressing waves. The reflection of time-

\footnotetext{
* The research in this paper was supported by the American Petroleum Institute (Project 61), the American Chemical Society (Petroleum Research Fund Grant in Aid 436-A) and the Office of Naval Research. Reproduction in whole or in part is permitted for any purpose of the United States Government.

† This work was done while Eliseu Resende was on leave of absence from the School of Engineering, University of Minas Gerais, Belo Horizonte, Brazil.
} 
independent progressing elastic waves from a rigid cylinder was considered by Resende [5] in an unpublished thesis, of which the present work is an extension. We shall utilize the methods and some results of references [1], [2] and [4] but nevertheless the present work can be read independently.

Progressing wave solutions of general hyperbolic equations have been discussed by Courant-Hilbert [6] and Ludwig [7], and have been studied in acoustics by Friedlander [8], [9]. Special reflection problems for elastic waves have been considered by various authors. In particular Gilbert [10] determined the leading term (i.e. the geometrical optics term) in the reflection of a time harmonic wave from a rigid or free cylinder.

In the final section we discuss our results and the use of the present method to solve other problems.

1. Progressing waves. In a homogeneous isotropic medium the displacement vector $\mathbf{u}$ can be written in terms of a scalar potential $v_{1}$ and a vector potential $\nabla_{2}$ in the form

$$
\mathbf{u}=\nabla v_{1}+\nabla \times \mathbf{v}_{2}
$$

The potentials satisfy the wave equations

$$
L_{j} v_{j}=0, \quad j=1,2 .
$$

Here $L_{i}$ and the propagation speed $c_{i}$ are defined by

$$
L_{i}=\Delta-c_{i}^{-2} \partial_{t}^{2}, \quad c_{1}^{2}=(\lambda+2 \mu) / \rho, \quad c_{2}^{2}=\mu / \rho .
$$

The constants $\rho, \lambda$ and $\mu$ are the density and the two Lamé constants of the medium respectively.

A progressing wave is a solution $v_{j}$ of (1.2) of the form

$$
v_{i}(t, \mathbf{x})=\sum_{m=0}^{\infty} e_{j}^{m}\left[s_{i}(t, \mathbf{x})\right] z_{j}^{m}(t, \mathbf{x})
$$

The functions $e_{i}^{m}$ are assumed to be related by

$$
e_{i}^{m}(s)=\frac{d}{d s} e_{i}^{m+1}(s) .
$$

Thus they are all iterated integrals of $e_{i}^{0}(s)$, which determines the wave form. Upon substituting (1.4) into (1.2) and using (1.5), we find that (1.2) is satisfied provided the phase function $s_{i}$ and the amplitude functions $z_{j}^{m}$ satisfy the equations

$$
\begin{aligned}
\left(\nabla s_{j}\right)^{2}-c_{i}^{-2}\left(\partial_{t} s_{i}\right)^{2} & =0 \\
2\left[\nabla s_{j} \cdot \nabla z_{j}^{m}-c_{j}^{-2}\left(\partial_{t} s_{j}\right)\left(\partial_{t} z_{j}^{m}\right)\right]+z_{j}^{m} L_{j} s_{j} & =-L_{j} z_{j}^{m-1}, \\
& m=0,1,2, \cdots ; \quad z_{j}^{-1} \equiv 0 .
\end{aligned}
$$

Eq. (1.6) is the characteristic equation of (1.2), and (1.7) are the transport equations. 
These equations can be solved by means of the characteristic curves of (1.6) which are also called the bicharacteristics of (1.2). They are straight lines which can be written in terms of a parameter $\sigma$ in the form

$$
\begin{aligned}
t & =\tilde{t}+c_{i}^{-1}(\sigma-\tilde{\sigma}) \\
\mathbf{x} & =\tilde{\mathbf{x}}+\mathbf{U}_{j}(\sigma-\tilde{\sigma}), \quad \mathbf{U}_{i}^{2}=1 .
\end{aligned}
$$

Here $\tilde{t}, \tilde{x}, \tilde{\sigma}$ and the unit vector $\mathrm{U}_{i}$ are constants on each bicharacteristic. The straight lines (1.9) in $\mathbf{x}$ space are called rays. Both (1.6) and (1.7) can be written as linear ordinary differential equations along bicharacteristics and therefore they can be solved explicitly with the results [2]

$$
\begin{aligned}
& s_{j}[t(\sigma), \mathbf{x}(\sigma)]=s_{j}[\tilde{t}, \tilde{\mathbf{x}}] \\
z_{i}^{m}[t(\sigma), \mathbf{x}(\sigma)]= & {\left[\frac{\left(\tilde{\sigma}+\rho_{j 1}\right)\left(\tilde{\sigma}+\rho_{i 2}\right)}{\left(\sigma+\rho_{i 1}\right)\left(\sigma+\rho_{i 2}\right)}\right]^{1 / 2} z_{i}^{m}(\tilde{\sigma}) } \\
& +\frac{c_{j}}{2 \partial_{t} s_{j}(\tilde{\sigma})} \int_{\tilde{\sigma}}^{\sigma}\left[\frac{\left(\sigma^{\prime}+\rho_{i 1}\right)\left(\sigma^{\prime}+\rho_{i 2}\right)}{\left(\sigma+\rho_{i 1}\right)\left(\sigma+\rho_{i 2}\right)}\right]^{1 / 2} L_{j} z_{j}^{m-1}\left(\sigma^{\prime}\right) d \sigma^{\prime}, \\
& m=0,1,2, \cdots .
\end{aligned}
$$

In these equations $t(\sigma)$ and $\mathbf{x}(\sigma)$ denote the right sides of (1.8) and (1.9) and we have written $z_{j}^{m}(\sigma)$ to denote $z_{j}^{m}[t(\tilde{\sigma}), \mathbf{x}(\tilde{\sigma})]$. In (1.11), $\rho_{i 1}$ and $\rho_{i 2}$, which are independent of $\sigma$, are defined in terms of a three parameter family of bicharacteristics $t\left(\sigma, \sigma_{1}, \sigma_{2}, \sigma_{3}\right), \mathbf{x}\left(\sigma, \sigma_{1}, \sigma_{2}, \sigma_{3}\right)$ with parameters $\sigma_{1}, \sigma_{2}, \sigma_{3}$. The Jacobian of the transformation from $\sigma, \sigma_{1}, \sigma_{2}, \sigma_{3}$ to $t, \mathbf{x}$ is, with $a_{i}$ independent of $\sigma$,

$$
\partial(t, \mathbf{x}) / \partial\left(\sigma, \sigma_{1}, \sigma_{2}, \sigma_{3}\right)=a_{i}^{-1}\left(\sigma+\rho_{i 1}\right)\left(\sigma+\rho_{i 2}\right) .
$$

These quantities $\rho_{i 1}$ and $\rho_{i 2}$ are the principal radii of curvature of the wavefront $s_{i}(\tilde{t}, \mathbf{x})=$ constant at the point $\sigma=0$ on the ray. In two space dimensions one radius, say $\rho_{i 2}$, may be taken to be infinite and the other can be denoted by $\rho_{i}$. In this case a two parameter family of bicharacteristics is used to define $\rho_{i}$. The initial values $s_{i}(\sigma)$ and $z_{j}^{m}(\sigma)$ on each bicharacteristic are arbitrary. The wave $v_{i}$ is given by (1.4) with $s_{i}$ given by (1.10) and $z_{i}^{m}$ by (1.11).

We shall now specialize the preceding results to a wave in two space dimensions. We represent the bicharacteristics by (1.8) and (1.9) with $\tilde{\sigma}=0, \tilde{\mathbf{x}}=$ $\mathrm{y}(\eta), \tilde{t}=\tau$, and $\mathrm{U}_{i}=\mathrm{U}_{i}(\tau, \eta)$. Then $\sigma$ denotes distance along a ray measured from the fixed curve $\mathrm{y}(\eta)$ and (1.8), and (1.9) become

$$
t=\tau+c_{j}^{-1} \sigma, \quad \mathrm{x}=y(\eta)+\sigma \mathrm{U}_{i}(\tau, \eta) .
$$

This is a two parameter family of bicharacteristics with $\tau$ and $\eta$ as the parameters. We choose $\eta$ to be arclength along the curve $\mathrm{y}(\eta)$ so that $(d \mathrm{y} / d \eta)^{2}=1$. We next define the angle $\phi_{i}(\tau, \eta)$ between the normal to the curve $\mathrm{y}(\eta)$ and the unit vector $\mathrm{U}_{i}(\tau, \eta)$ along a ray by

$$
\mathrm{U}_{i}(\tau, \eta) \cdot \frac{d \mathrm{y}(\eta)}{d \eta}=\sin \phi_{i}(\tau, \eta)
$$


Then the Jacobian of the transformation (1.13) from $\sigma, \tau, \eta$ to $t, \mathrm{x}$ can be written in the form

$$
\frac{\partial(t, \mathbf{x})}{\partial(\sigma, \tau, \eta)}=a_{i}^{-1}\left(\sigma+\rho_{i}\right)
$$

Now $a_{i}$ and the radius of curvature $\rho_{i}$ are given in terms of $\phi$ and the signed curvature $\kappa(\eta)$ of the curve y $(\eta)$ by

$$
a_{i}^{-1}=\partial_{\eta} \phi_{i}+\kappa(\eta)+c_{i}^{-1}\left(\partial_{\tau} \phi_{i}\right) \sin \phi_{i}, \quad \rho_{i}=a_{i} \cos \phi_{i} .
$$

If $\theta(\eta)$ is the angle between the tangent $d \mathrm{y}(\eta) / d \eta$ to the curve $\mathrm{y}(\eta)$ and the positive $x_{1}$-axis, then $\kappa(\eta)=d \theta(\eta) / d \eta$.

In the two dimensional case (1.11) becomes

$$
z_{j}^{m}(\sigma)=\left[\frac{\rho_{i}}{\sigma+\rho_{i}}\right]^{1 / 2} z_{i}^{m}(0)+\frac{c_{j}}{2 \partial_{t} s_{j}(0)} \int_{0}^{\sigma}\left[\frac{\sigma^{\prime}+\rho_{i}}{\sigma+\rho_{i}}\right]^{1 / 2} L_{j} z_{i}^{m-1}\left(\sigma^{\prime}\right) d \sigma^{\prime} .
$$

We shall now prove by induction that in two dimensions $z_{i}^{m}(\sigma)$, given by (1.17), has the form

$$
z_{i}^{m}(\sigma)=\sum_{a=0}^{3 m} F_{j a}^{m}(\tau, \eta)\left[\sigma+\rho_{i}(\tau, \eta)\right]^{-\alpha-1 / 2}
$$

For $m=0$ this follows from (1.17), since $z_{j}^{-1}=0$ by definition. We now assume that (1.18) holds for $m-1$ and use it in the integrand in (1.17). We use for the operator $L_{i}$ its expression in terms of $\sigma, \tau$ and $\eta$, given by (A.3) of the appendix. Then we find that $z_{j}^{m}(\sigma)$ is indeed given by (1.18) which completes the induction. At the same time we obtain a recursion formula for the coefficients $F_{j a}^{m}(\tau, \eta)$ in (1.18), which determines them in terms of $F_{j 0}^{0}(\tau, \eta)$.

We shall omit it because it is lengthy. The special case of this recursion formula for time-independent progressing waves will be given in Section 3 . Now the wave $v_{i}$ is given by (1.4) with $s_{j}$ given by (1.10) and $z_{i}^{m}$ given (1.18). In terms of the parameters $\sigma, \tau, \eta$ these waves can be written

$$
v_{j}(t, \mathbf{x})=\sum_{m=0}^{\infty} e_{i}^{m}\left[s_{j}(\tau, \mathrm{y}(\eta))\right] \sum_{\alpha=0}^{3 m} F_{i q}^{m}(\tau, \eta)\left[\sigma+\rho_{j}(\tau, \eta)\right]^{-a-1 / 2} .
$$

Here $t$ and $\mathbf{x}$ are given by (1.13).

2. Reflection of progressing waves. Let $\mathbf{x}=\mathbf{y}(\eta)$ be the equation of a stationary rigid cylindrical surface $C$ upon which is incident the two dimensional progressing wave

$$
v_{0}(t, \mathbf{x})=\sum_{m=0}^{\infty} e_{0}^{m}\left[s_{0}(t, x)\right] z_{0}^{m}(t, \mathbf{x}) .
$$

We shall write the reflected field in the form (1.1) with $v_{1}$ and $v_{2}$ given by (1.4). Then we shall adjust the arbitrary quantities in these progressing waves so that the total displacement vanishes on the cylinder. For definiteness we shall assume 
that the incident wave (2.1) is a compressional wave, i.e. a wave like $v_{1}$ in Section 1 , although the case of an incident shear wave can be treated in a very similar way. In the rest of this work, $\mathbf{x}=\left(x_{1}, x_{2}\right)$ denotes a point in two dimensional space.

The reflected field will have no displacement in the $x_{3}$ direction, as we can see from symmetry, so the vector potential will have only an $x_{3}$ component. Therefore we shall write $\nabla_{2}=\left(0,0, v_{2}\right)$. Then the total displacement $\mathfrak{u}$ is

$$
\mathbf{u}=\left[\partial_{x_{1}}\left(v_{0}+v_{1}\right)+\partial_{x_{2}} v_{2}, \partial_{x_{2}}\left(v_{0}+v_{1}\right)-\partial_{x_{1}} v_{2}\right] \text {. }
$$

Now the boundary condition of no displacement on the cylinder becomes

$$
\begin{array}{lll}
\partial_{x_{1}}\left(v_{0}+v_{1}\right)+\partial_{x_{2}} v_{2}=0 & \text { on } C, \\
\partial_{x_{2}}\left(v_{0}+v_{1}\right)-\partial_{x_{1}} v_{2}=0 & \text { on } C .
\end{array}
$$

We next substitute (1.4) and (2.1) for $v_{1}, v_{2}$ and $v_{0}$ into (2.3) and (2.4). We find that (2.3) and (2.4) will be satisfied provided that the following equations hold:

$$
\begin{gathered}
e_{1}^{m}=e_{2}^{m}=e_{0}^{m}, \quad m=0,1, \cdots, \\
s_{1}[t, \mathrm{y}(\eta)]=s_{2}[t, \mathrm{y}(\eta)]=s_{0}[t, \mathrm{y}(\eta)] . \\
z_{1}^{m} \partial_{x_{1}} s_{1}+z_{2}^{m} \partial_{x_{2}} s_{2}=-z_{0}^{m} \partial_{x_{1}} s_{0}-\partial_{x_{1}} z_{0}^{m-1}-\partial_{x_{1}} z_{1}^{m-1}-\partial_{x_{2}} z_{2}^{m-1} \quad \text { on } C, \\
m=0,1, \cdots, \\
z_{1}^{m} \partial_{x_{2}} s_{1}-z_{2}^{m} \partial_{x_{2}} s_{2}=-z_{0}^{m} \partial_{x_{2}} s_{0}-\partial_{x_{2}} z_{0}^{m-1}-\partial_{x_{2}} z_{1}^{m-1}-\partial_{x_{2}} z_{2}^{m-1} \quad \text { on } C, \\
m=0,1, \cdots .
\end{gathered}
$$

Differentiating (2.6) with respect to $t$ and with respect to $\eta$ yields

$$
\begin{gathered}
\partial_{t} s_{1}=\partial_{t} s_{2} \quad \text { on } C . \\
\nabla s_{1} \cdot \frac{d \mathrm{y}}{d \eta}=\nabla s_{2} \cdot \frac{d \mathrm{y}}{d \eta}=\nabla s_{0} \cdot \frac{d \mathrm{y}}{d \eta} \text { on } C .
\end{gathered}
$$

It follows from the theory of characteristics applied to (1.6) that the ray direction $\mathrm{U}_{j}$ is the direction of $\nabla s_{i}$ for $j=1$ and $j=2$ and also for $j=0$, since the wave $v_{0}$ is of the same type as $v_{1}$. The length of $\nabla s_{1}$ is $c_{i}^{-1}\left|\partial_{t} s_{j}\right|$ as we see from (1.6), so we have

$$
\nabla s_{i}=c_{j}^{-1}\left|\partial_{t} s_{j}\right| \mathrm{U}_{j} .
$$

We now use (2.11) for $\nabla s_{i}$ in (2.10) and make use of (2.9) to obtain

$$
c_{1}^{-1} \mathrm{U}_{1} \cdot \frac{d \mathrm{y}}{d \eta}=c_{2}^{-1} \mathrm{U}_{2} \cdot \frac{d \mathrm{y}}{d \eta}=c_{1}^{-1} \mathrm{U}_{0} \cdot \frac{d \mathrm{y}}{d \eta} \text { on } C .
$$

Upon introducing $\phi_{i}$ defined by (1.14), we obtain from (2.12) the two equations

$$
\sin \phi_{1}=\sin \phi_{0}, \quad c_{2}^{-1} \sin \phi_{2}=c_{1}^{-1} \sin \phi_{0} .
$$


These are the laws of reflection of elasticity. From the definitions of $\phi_{i}$, if we choose the normal pointing into the cylinder, then $\pi / 2<\phi_{1}<\pi, \pi / 2<\phi_{2}<\pi$ and $0<\phi_{0}<\pi / 2$. (See Figure 1.) It follows from (2.13) that

$$
\phi_{1}=\pi-\phi_{0}, \quad \phi_{2}=\pi-\sin ^{-1}\left[\left(\frac{\mu}{\lambda+2 \mu}\right)^{1 / 2} \sin \phi_{0}\right] .
$$

These results determine the direction of the reflected bicharacteristics and of their projections into $x$-space, the reflected rays. Then $s_{1}$ and $s_{2}$ are constant along these bicharacteristics with the constant value $s_{0}[t, \mathrm{y}(\eta)]$ given by (2.6). In this way $s_{1}$ and $s_{2}$ are determined at all points of the reflected waves.

Let $\theta(\eta)$ be the previously defined angle between $d \mathrm{y} / d \eta$, the tangent to $C$, and the positive $x_{1}$ axis. Then we can write $\mathrm{U}_{i}$ in the form

$$
\mathrm{U}_{i}=\left[\sin \left(\phi_{i}+\theta\right),-\cos \left(\phi_{i}+\theta\right)\right] \text {. }
$$

By using (2.9) and (2.15), we can write (2.11) in the form

$$
\nabla s_{i}=c_{i}^{-1}\left|\partial_{t} s_{0}\right|\left[\sin \left(\phi_{i}+\theta\right),-\cos \left(\phi_{i}+\theta\right)\right] \text {. }
$$

Now we can solve (2.7) and (2.8) for $z_{i}^{m}(0)$, using (2.16) to eliminate $\nabla s_{i}$ and the

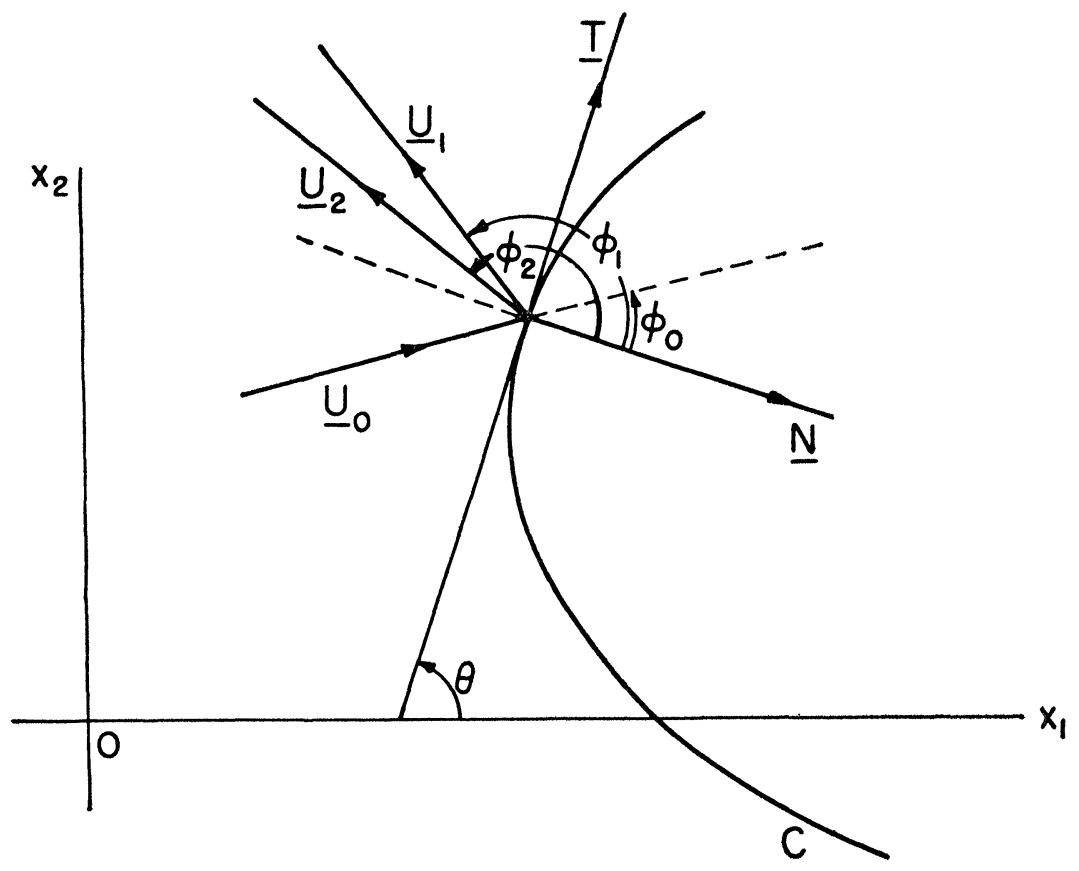

Figure 1. An incident ray in the direction $U_{0}$ is shown hitting the curve $C$ which bounds the cross section of a reflecting cylinder. The reflected compressional ray in the direction ${U_{1}}_{1}$ and the reflected shear ray in the direction $\mathrm{U}_{2}$ are indicated, as are the directions of the $x_{1}$ and $x_{2}$ axes. The angle between the tangent $\mathrm{T}$ to $\mathrm{C}$ and the positive $x_{1}$-axis is denoted by $\theta$. 
first equation in (2.14) to eliminate $\phi_{2}$. Then we obtain

$$
\begin{aligned}
z_{1}^{m}(0)= & R_{1} z_{0}^{m}(0)+c_{1}\left[\left|\partial_{t} s_{0}\right| \cos \left(\phi_{2}+\phi_{0}\right)\right]^{-1} \\
& \cdot\left[\left(\partial_{x_{2}} z_{0}^{m-1}+\partial_{x_{2}} z_{1}^{m-1}-\partial_{x_{1}} z_{2}^{m-1}\right) \cos \left(\phi_{2}+\theta\right)\right. \\
& \left.\quad-\left(\partial_{x_{1}} z_{0}^{m-1}+\partial_{x_{1}} z_{1}^{m-1}+\partial_{x_{2}} z_{2}^{m-1}\right) \sin \left(\phi_{2}+\theta\right)\right], \\
z_{2}^{m}(0)= & R_{2} z_{0}^{m}(0)+c_{2}\left[\left|\partial_{t} s_{0}\right| \cos \left(\phi_{2}+\phi_{0}\right)\right]^{-1} \\
& \cdot\left[\left(\partial_{x_{2}} z_{0}^{m-1}+\partial_{x_{2}} z_{1}^{m-1}-\partial_{x_{1}} z_{2}^{m-1}\right) \sin \left(\phi_{0}-\theta\right)\right. \\
& \left.-\left(\partial_{x_{1}} z_{0}^{m-1}+\partial_{x_{1}} z_{1}^{m-1}+\partial_{x_{2}} z_{2}^{m-1}\right) \cos \left(\phi_{0}-\theta\right)\right] .
\end{aligned}
$$

Here the reflection coefficients $R_{i}$ are defined by

$$
R_{1}=\frac{\cos \left(\phi_{2}-\phi_{0}\right)}{\cos \left(\phi_{2}+\phi_{0}\right)}, \quad R_{2}=\frac{c_{2} \sin 2 \phi_{0}}{c_{1} \cos \left(\phi_{2}+\phi_{0}\right)} .
$$

By using $z_{j}^{m}(0)$ given by (2.17) and (2.18) in (1.17), we obtain the $z_{i}^{m}(\sigma)$ at all points of the reflected waves. For $m=0$, we find

$$
z_{j}^{0}(\sigma)=\left[\frac{\rho_{i}}{\sigma+\rho_{j}}\right]^{1 / 2} R_{j} z_{0}^{0}(0)
$$

Then (1.18) and (2.20) yield

$$
F_{i 0}^{0}(\tau, \eta)=\rho_{i}^{1 / 2} R_{i} z_{0}^{0}(0) .
$$

The other $F_{i \alpha}^{m}$ can be found recursively from $F_{i 0}^{0}$, given by (2.21).

We can now write the reflected potentials in the form (1.19) with $e_{i}^{m}\left[s_{i}(\tau, \mathbf{y}(\eta))\right]$ replaced by $e_{0}^{m}\left[s_{0}(\tau, \mathrm{y}[\eta])\right]$. The reflected bicharacteristics are giv an by (1.13) with $\mathrm{U}_{i}$ given by $(2.15)$.

3. Reflection of time-independent progressing waves. A progressing wave of the form (1.4) is called time-independent if $z_{j}^{m}$ and $s_{i}$ are of the form

$$
z_{j}^{m}(t, \mathbf{x})=z_{i}^{m}(\mathbf{x}), \quad s_{j}(t, \mathbf{x})=t-c_{j}^{-1} \psi_{j}(\mathbf{x}) .
$$

It follows from (3.1) and (1.6) that $\psi_{i}$ satisfies the eiconal equation

$$
\left(\nabla \psi_{i}\right)^{2}=1 \text {. }
$$

From (1.10) and (3.1) it follows that along a bicharacteristic $\psi_{i}$ is given by

$$
\psi_{i}[\mathbf{x}(\sigma)]=\sigma-\tilde{\sigma}+\psi_{i}[\mathbf{x}(\tilde{\sigma})] .
$$

From (3.1) we have $\partial_{t} s_{j}=1$ and $L_{j} z_{j}^{m-1}=\Delta z_{i}^{m-1}$ so (1.11) and (1.17) become slightly simpler. Upon combining these results we find that on a bicharacteristic

$$
\begin{aligned}
v_{j}(t, \mathrm{x})= & \sum_{m=0}^{\infty} e_{j}^{m}\left[t-c_{i}^{-1}\left(\sigma-\tilde{\sigma}+\psi_{i}\{\mathrm{x}(\tilde{\sigma})\}\right)\right] \\
& \cdot\left\{[J(\tilde{\sigma}) / J(\sigma)]^{1 / 2} z_{j}^{m}(\sigma)+\frac{c_{j}}{2} \int_{\tilde{\sigma}}^{\sigma}\left[J\left(\sigma^{\prime}\right) / J(\sigma)\right]^{1 / 2} \Delta z_{j}^{m-1}\left(\sigma^{\prime}\right) d \sigma^{\prime}\right\} .
\end{aligned}
$$

The values of $t$ and $\mathbf{x}$ are given by (1.8) and (1.9). 
For a two dimensional time-independent progressing wave, (1.18) and (1.19) simplify because $F_{j q}^{m}$ is independent of $\tau$ and $s_{i}$ is given by (3.1). It is convenient to set $F_{i q}^{m}(\eta)=\left(c_{i}\right)^{m} f_{i \alpha}^{m}(\eta)$ and then (1.18) and (1.19) become

$$
\begin{gathered}
z_{j}^{m}(\sigma)=\left(c_{j}\right)^{m} \sum_{q=0}^{3 m} f_{j \alpha}^{m}(\eta)\left[\sigma+\rho_{j}(\eta)\right]^{-\alpha-1 / 2} \\
v_{j}(t, \mathbf{x})=\sum_{m=0}^{\infty}\left(c_{j}\right)^{m} e_{0}^{m}\left[t-c_{j}^{-1}\left(\sigma+\psi_{j}\{\mathrm{y}(\eta)\}\right)\right] \cdot \sum_{\alpha=0}^{3 m} f_{j \alpha}^{m}(\eta)\left[\sigma+\rho_{i}(\eta)\right]^{-\alpha-1 / 2} .
\end{gathered}
$$

In writing (3.6) we have made use of (2.5). The values of $t$ and $\mathbf{x}$ are given by (1.13). It also follows from (3.1) that $\mathrm{U}_{j}(\eta)$ is independent of $\tau$ and therefore $\phi_{i}(\eta), b_{j}(\eta)$ and $\rho_{j}(\eta)$ are also independent of $\tau$.

To obtain the recursion formula for the $f_{i \alpha}^{m}$ in (3.5) we insert $z_{i}^{m}$ into (1.17). Upon using the facts that $\partial_{j} s_{j}=1$ and $L_{j} z_{j}^{m-1}=\Delta z_{j}^{m-1}$, and the representation of $\Delta$ in (A.10) we obtain

$$
\begin{array}{r}
f_{j a}^{m}=\frac{1}{2 q}\left[a_{j} \partial_{\eta}\left(a_{j} \partial_{\eta} f_{i, q-1}^{m-1}\right)+2(q-1) b_{j} a_{i} \partial_{\eta} f_{i, q-2}^{m-1}+(q-1 / 2)^{2} f_{j, q-1}^{m-1}\right. \\
\left.+(q-3 / 2) a_{j}\left(\partial_{\eta} b_{j}\right) f_{i, q-2}^{m-1}+b_{i}^{2}(q-5 / 2)(q-1 / 2) f_{i, q-3}^{m-1}\right], \\
q, m=1,2, \cdots ; \quad j=1,2 .
\end{array}
$$

Here $b_{j}$ is defined by

$$
b_{j}=a_{j}\left(\sin \phi_{j}-\partial_{\eta} \rho_{j}\right)
$$

In addition

$$
f_{i q}^{m}=0 \text { for } q<0, \quad q>3 m .
$$

The result (3.5) and the recursion formulas (3.7)-(3.9) were obtained by Keller, Lewis and Seckler [4] who used slightly different independent variables. To obtain (3.7) from their (18.4) we must replace their $\partial_{\theta}$ by $a_{i} \partial_{\eta}$ and their $b$ by our $b_{j}$.

Since (3.7) does not hold for $q=0$, we set $\sigma=0$ in (3.5) and solve the resulting equation for $f_{j 0}^{m}$ to obtain

$$
f_{j 0}^{m}(\eta)=\left(c_{j}\right)^{-m} \rho_{i}^{1 / 2} z_{i}^{m}(0, \eta)-\sum_{\alpha=1}^{3 m} f_{j \alpha}^{m}(\eta) \rho_{j}^{-a}(\eta) .
$$

Now $z_{j}^{m}(0)$ is given by (2.17) and (2.18) in which $\partial_{t} s_{j}=1$. However these equations involve $\partial_{x_{1}} z_{j}^{m-1}(0)$ and $\partial_{x_{2}} z_{j}^{m-1}(0)$, which we wish to replace by expressions involving $f_{j a}^{m-1}$. To this end we differentiate the expression (3.5) for $z_{j}^{m}(\sigma)$ with respect to $x_{\eta}, i=1,2$ and then set $\sigma=0$ to get

$$
\begin{aligned}
\partial_{x_{i}} z_{i}^{m}(0)=-\left(\partial_{x_{i}} \sigma\right) & \sum_{q=0}^{3 m}(q+1 / 2) f_{j \alpha}^{m} \rho_{j}^{-\alpha-3 / 2} \\
+\left(\partial_{x_{i}} \eta\right) & \sum_{q=0}^{3 m}\left[\rho_{j}^{-\alpha-1 / 2} \partial_{\eta} f_{i \alpha}^{m}-(q+1 / 2) f_{i \alpha}^{m} \rho_{i}^{-\alpha-3 / 2} \partial_{\eta} \rho_{i}\right]
\end{aligned}
$$


The derivatives $\partial_{x_{i}} \sigma$ and $\partial_{x_{i}} \eta$ can be obtained from the ray equation (1.13) in which $\mathrm{U}_{j}$ is given by (2.15). Since this equation involves $j$, the derivatives depend upon $j$. These derivatives are calculated in the appendix and are given by (A.4), (A.5), (A.7) and (A.8) in which we must set $\sigma=0$ and $\partial_{r} \phi=0$. Then we find

$$
\begin{aligned}
& \partial_{x_{1}} \sigma=\sin \left(\phi_{j}+\theta\right)-a_{j} \rho_{j}^{-1} \cos \left(\phi_{j}+\theta\right) \sin \phi_{j}, \\
& \partial_{x_{2}} \sigma=-\cos \left(\phi_{i}+\theta\right)-a_{j} \rho_{j}^{-1} \sin \left(\phi_{j}+\theta\right) \sin \phi_{j}, \\
& \partial_{x_{1}} \eta=a_{j} \rho_{j}^{-1} \cos \left(\phi_{j}+\theta\right), \quad \partial_{x_{2}} \eta=a_{j} \rho_{j}^{-1} \sin \left(\phi_{j}+\theta\right) .
\end{aligned}
$$

We now use (3.12)-(3.14) in (3.11) to obtain $\partial_{x_{i}} z_{i}^{m}(0)$ in terms of $f_{i q}^{m}, q=0$, $\cdots, 3 m$. Then we use $\partial_{x_{i}} z_{i}^{m-1}(0)$ in (2.17) and (2.18) to obtain

$$
\begin{aligned}
z_{1}^{m}(0) & =R_{1} z_{0}^{m}(0)+c_{1} \sec \left(\phi_{2}+\phi_{0}\right) \\
\cdot & \left\{-\partial_{\sigma_{2}} z_{0}^{m-1}+A_{1}^{m-1} \cos \left(\phi_{2}-\phi_{1}\right)-B_{1}^{m-1} \sin \left(\phi_{2}-\phi_{1}\right)-B_{2}^{m-1}\right\} \\
z_{2}^{m}(0)= & R_{2} z_{0}^{m}(0)+c_{2} \sec \left(\phi_{2}+\phi_{0}\right) \\
& \cdot\left\{\partial_{\nu_{1}} z_{0}^{m-1}-A_{2}^{m-1} \cos \left(\phi_{2}+\phi_{0}\right)-B_{2}^{m-1} \sin \left(\phi_{2}+\phi_{0}\right)+B_{1}^{m-1}\right\}
\end{aligned}
$$

The quantities $A_{i}^{m-1}$ and $B_{i}^{m-1}$ are defined by

$$
\begin{aligned}
& A_{i}^{m-1}(\eta)=\sum_{q=0}^{3(m-1)}(q+1 / 2) f_{j a}^{m-1} \rho_{j}^{-q-3 / 2}, \\
& B_{i}^{m-1}(\eta)=\sum_{q=0}^{3(m-1)}(q+1 / 2) f_{j a}^{m-1} \rho_{j}^{-q-5 / 2}+a \sum_{q=0}^{3(m-1)} \rho_{i}^{-a-3 / 2} \partial_{\eta} f_{i q}^{m-1} .
\end{aligned}
$$

In addition $A_{i}^{-1}=B_{i}^{-1}=0$. In (3.15) $\partial_{\sigma_{3}} z_{0}^{m-1}$ denotes the derivative of $z_{0}^{m-1}$ with respect to $\sigma$ along a reflected shear ray and in (3.16) $\partial_{\nu_{1}} z_{0}^{m-1}$ is the derivative of $z_{0}^{m-1}$ in the direction normal to a reflected compressional ray. In terms of $x_{1}$ and $x_{2}$ derivatives,

$$
\begin{aligned}
& \partial_{\sigma_{2}} z_{0}^{m-1}=\sin \left(\phi_{2}+\theta\right) \partial_{x_{1}} z_{0}^{m-1}-\cos \left(\phi_{2}+\theta\right) \partial_{x_{2}} z_{0}^{m-1} \\
& \partial_{\nu_{1}} z_{0}^{m-1}=\cos \left(\phi_{1}+\theta\right) \partial_{x_{1}} z_{0}^{m-1}+\sin \left(\phi_{1}+\theta\right) \partial_{x_{2}} z_{0}^{m-1} .
\end{aligned}
$$

The $f_{i q}^{m}$ can now be obtained recursively by using (3.7) for $q, m=1,2, \cdots$ and (3.10) for $q=0$ with $z_{i}^{m}(0)$ given by (3.15)-(3.18). Then $z_{i}^{m}(\sigma)$ is given by (3.5), $v_{i}(t, \mathrm{x})$ is given by (3.6) and $\mathrm{x}$ is given by (1.13) in which $\mathrm{U}_{i}(\eta)$ is given by (2.15). The total displacement $\mathbf{u}$ is now given by (2.2) in which $v_{0}$ is given by (2.1) while $v_{1}$ and $v_{2}$ are given by (3.6) in the manner just described.

To express these potentials at a point $\mathrm{x}$ it is convenient to denote by $\sigma_{1}$ and $\eta_{1}$ the values of $\sigma$ and $\eta$ on a reflected compressional ray at $\mathrm{x}$ and by $\sigma_{2}$ and $\eta_{2}$ the corresponding quantities on a reflected shear ray at $\mathrm{x}$. Thus $\mathrm{y}\left(\eta_{i}\right)$ is the point of reflection of a ray of type $j$ which passes through $\mathrm{x}$ and $\sigma_{j}$ is the distance along this ray from $\mathrm{y}\left(\eta_{i}\right)$ to $\mathrm{x}$. Then we can write 


$$
\begin{gathered}
v_{0}(t, \mathbf{x})+v_{1}(t, \mathbf{x})=\sum_{m=0}^{\infty}\left(c_{2}\right)^{m} e_{0}^{m}\left[t-c_{2}^{-1} \psi_{0}(\mathbf{x})\right] z_{0}^{m}(\mathbf{x}) \\
+\sum_{m=0}^{\infty}\left(c_{1}\right)^{m} e_{0}^{m}\left[t-c_{1}^{-1}\left\{\psi_{0}[\mathrm{y}(\eta)]+\sigma_{1}\right\}\right] \sum_{q=0}^{3 m} f_{1 q}^{m}\left(\eta_{1}\right)\left[\sigma_{1}+\rho_{1}\left(\eta_{1}\right)\right]^{-a-1 / 2} \\
v_{2}(t, \mathbf{x})=\sum_{m=0}^{\infty}\left(c_{2}\right)^{m} e_{0}^{m}\left[t-c_{2}^{-1}\left\{\psi_{0}\left[\mathrm{y}\left(\eta_{2}\right)+\sigma_{2}\right\}\right] \sum_{q=0}^{3 m} f_{2 q}^{m}\left(\eta_{2}\right)\left[\sigma_{2}+\rho_{2}\left(\eta_{2}\right)\right]^{-q-1 / 2}\right.
\end{gathered}
$$

If there are more than one reflected ray of either type through $\mathbf{x}$, we must sum over the corresponding values of $\sigma_{1}$ and $\eta_{1}$ in (3.21) and over the values of $\sigma_{2}$ and $\eta_{2}$ in (3.22).

As an illustration of the preceding method, we shall consider the incident plane compressional wave with potential $v_{0}$ given by

$$
v_{0}(t, x)=e_{0}^{0}\left(t-c_{1}^{-1} x_{1}\right) .
$$

This is an exact solution of the wave equation $L_{1} v_{0}=0$ for any $e_{0}^{0}$. From (3.23) we see that $v_{0}$ represents a time-independent progressing wave with

$$
\psi_{0}(\mathbf{x})=x_{1}, \quad z_{0}^{m}(\mathbf{x})=\delta_{m 0} .
$$

The unit vector $\mathrm{U}_{0}$ in the ray direction is just $\nabla \psi_{0}=(1,0)$, which is along the positive $x_{1}$ axis. Thus (1.14) yields $\sin \phi_{0}(\eta)=d y_{1}(\eta) / d \eta$. On the other hand from the definition of $\theta(\eta), \cos \theta(\eta)=d y_{1} / d \eta$. Therefore $\sin \phi_{0}=\cos \theta$ so $\phi_{0}=\pi / 2-\theta$. Differentiating this relation and recalling that $d \theta / d \eta=\kappa$ yields

$$
-d \phi_{0} / d \eta=\kappa(\eta)
$$

Then (2.14) and (3.25) yield

$$
\frac{d \phi_{1}}{d \eta}=\kappa, \quad \frac{d \phi_{2}}{d \eta}=-\kappa \frac{c_{2} \cos \phi_{0}}{c_{1} \cos \phi_{2}}
$$

Upon using (3.26) in (1.16) we obtain

$$
a_{1}=(2 k)^{-1}, \quad a_{2}=c_{1} \cos \phi_{2}\left[k\left(c_{1} \cos \phi_{2}-c_{2} \cos \phi_{0}\right)\right]^{-1} .
$$

Let us now calculate $z_{i}^{m}(0)$ from (3.15) and (3.16). For $m=0$, (3.24) yields $z_{0}^{0}=1$ and since $z_{0}^{-1}=A_{j}^{-1}=B_{j}^{-1}=0$, we obtain

$$
z_{j}^{0}(0)=R_{i} \text {. }
$$

For $m>0, z_{0}^{m}=0$ and $\partial_{\sigma_{2}} z_{0}^{m-1}=\partial_{\nu_{1}} z_{0}^{m-1}=0$ so (3.15) and (3.16) become

$$
\begin{aligned}
z_{1}^{m}(0)=c_{1} \sec \left(\phi_{2}+\phi_{0}\right) & \\
& \cdot\left\{A_{1}^{m-1} \cos \left(\phi_{2}-\phi_{1}\right)-B_{1}^{m-1} \sin \left(\phi_{2}-\phi_{1}\right)-B_{2}^{m-1}\right\} \\
z_{2}^{m}(0)=c_{2} \sec \left(\phi_{2}+\phi_{0}\right) & \\
\cdot & \left\{-A_{2}^{m-1} \cos \left(\phi_{2}+\phi_{0}\right)-B_{2}^{m-1} \sin \left(\phi_{2}+\phi_{0}\right)+B_{1}^{m-1}\right\}
\end{aligned}
$$


By using (1.16) we can replace $\rho_{i}$ by $a_{i} \cos \phi_{i}$ in these equations and use (3.27) for $a_{i}$. To complete the determination of the solution we proceed in the manner described after (3.20).

4. Discussion. We have seen that in homogeneous isotropic media, elastic waves can be represented by progressing wave expansions, which are formal solutions of the linear equations of elasticity. Although they are infinite expansions in general, they are most useful when the leading term, or a few terms, suffice to describe a wave accurately. This is the case when the wave consists mainly of components with wavelengths short compared to the radii of curvature of the wavefronts. The leading term of the wave (1.4) on a bicharacteristic (1.8) and (1.9) is

$$
\begin{aligned}
v_{j}\left[\tilde{t}+c_{i}^{-1}(\sigma-\tilde{\sigma}), \tilde{\mathbf{x}}+\mathrm{U}_{j}(\sigma-\tilde{\sigma})\right] & \\
& \sim e_{0}^{0}\left[s_{i}(\tilde{t}, \tilde{x})\right]\left[\frac{\left(\tilde{\sigma}+\rho_{i 1}\right)\left(\tilde{\sigma}+\rho_{i 2}\right)}{\left(\sigma+\rho_{i 1}\right)\left(\sigma+\rho_{i 2}\right)}\right]^{1 / 2} z_{i}^{0}(\tilde{t}, \tilde{\mathbf{x}}) .
\end{aligned}
$$

If we measure $\sigma$ along a ray from $\tilde{\mathbf{x}}$ then $\tilde{\sigma}=0$ and (4.1) becomes

$$
v_{j}\left[t+c_{j}^{-1} \sigma, \tilde{\mathbf{x}}+\mathbf{U}_{j} \sigma\right] \sim e_{0}^{0}\left[s_{j}(\tilde{t}, \tilde{\mathbf{x}})\right]\left[\frac{\rho_{i 1} \rho_{i 2}}{\left(\sigma+\rho_{i 1}\right)\left(\sigma+\rho_{i 2}\right)}\right]^{1 / 2} z_{j}^{0}(\tilde{t}, \tilde{\mathbf{x}}) .
$$

In two dimensions (4.2) becomes

$$
v_{j}\left[\tilde{t}+c_{i}^{-1} \sigma, \tilde{\mathbf{x}}+\mathrm{U}_{i} \sigma\right] \sim e_{0}^{0}\left[s_{j}(\tilde{t}, \tilde{\mathbf{x}})\right]\left[\rho_{j} /\left(\sigma+\rho_{i}\right)\right]^{1 / 2} z_{j}^{0}(\tilde{t}, \tilde{\mathbf{x}}) .
$$

For the reflected waves in two dimensions, $z_{j}^{0}$ is given by (2.20) provided $\sigma=0$ at the reflector. When that result is used, (4.3) becomes

$$
v_{i}\left[\tau+c_{i}^{-1} \sigma, \mathrm{y}(\eta)+\mathrm{U}_{i} \sigma\right] \sim e_{0}^{0}\left[s_{0}(\tau, \mathrm{y}(\eta))\right]\left[\rho_{i} /\left(\sigma+\rho_{i}\right)\right]^{1 / 2} R_{j} z_{0}^{0}[\tau, \mathrm{y}(\eta)]
$$

Completely analogous results can be obtained in three dimensions and for reflection from free surfaces, interfaces, etc. (See, for example, H. B. Keller [11].)

The progressing wave expansion can also be used to solve various propagation, reflection, refraction, diffraction, radiation and other problems. (See, for example, Keller and Lewis [12].) It can also be improved upon to eliminate its failure at caustics by the introduction of boundary layers or by the uniform method of Ludwig [13].

Appendix. Expressions for $L_{i}$ and $\Delta$ in ray coordinates. In the case of two space dimensions, the bicharacteristics are given in terms of the ray coordinates $\tau, \sigma, \eta$ by (1.13). If in (1.13) we use (2.15) for $\mathrm{U}_{i}$ we obtain

$$
\begin{aligned}
x_{1} & =y_{1}(\eta)+\sigma \sin \left(\phi_{i}+\theta\right) \\
x_{2} & =y_{2}(\eta)-\sigma \cos \left(\phi_{i}+\theta\right) \\
t & =\tau+c_{i}^{-1} \sigma .
\end{aligned}
$$


We wish to express $L_{i}=\Delta-c_{i}^{-2} \partial_{t}^{2}$ in terms of ray coordinates. To this end we observe that

$$
\begin{aligned}
\partial_{x_{i}} & =\left(\partial_{x_{i}} \sigma\right) \partial_{\sigma}+\left(\partial_{x i} \tau\right) \partial_{\tau}+\left(\partial_{x_{i}} \eta\right) \partial_{\eta}, \quad i=1,2 \\
\partial_{t} & =\left(\partial_{t} \sigma\right) \partial_{\sigma}+\left(\partial_{t} \tau\right) \partial_{\tau}+\left(\partial_{t} \eta\right) \partial_{\eta} .
\end{aligned}
$$

Thus

$$
\begin{aligned}
L_{i}=\sum_{i=1}^{2}\left[\left(\partial_{x_{i}} \sigma\right) \partial_{\sigma}+\left(\partial_{x_{i}} \tau\right) \partial_{\tau}+\left(\partial_{x_{i}} \eta\right) \partial_{\eta}\right]^{2} & \\
& -c_{i}^{-2}\left[\left(\partial_{t} \sigma\right) \partial_{\sigma}+\left(\partial_{t} \tau\right) \partial_{\tau}+\left(\partial_{t} \eta\right) \partial_{\eta}\right]^{2} .
\end{aligned}
$$

To determine the coefficients in (A.3) we differentiate (A.1) with respect to $x_{i}, i=1,2$, and with respect to $t$ and solve the resulting equations to obtain

$$
\begin{aligned}
\partial_{x_{1}} \sigma=-c_{i} \partial_{x_{1}} \tau & =a_{j}\left(\partial_{\eta} \phi_{i}+\kappa\right) \sin \left(\phi_{i}+\theta\right)-\left(\sigma+\rho_{i}\right)^{-1} a_{i}^{2} \\
& \cdot\left[\left(\partial_{\eta} \phi_{j}+\kappa\right) \cos \left(\phi_{i}+\theta\right)-c_{i}^{-1} \partial_{\tau} \phi_{j} \sin \theta\right] \sin \phi_{i},
\end{aligned}
$$

$$
\begin{aligned}
& \partial_{x_{2}} \sigma=-c_{j} \partial_{x_{2}} \tau=a_{j}\left(\partial_{\eta} \phi_{i}+\kappa\right) \cos \left(\phi_{i}+\theta\right)+\left(\sigma+\rho_{i}\right)^{-1} a_{i}^{2} \\
& \cdot\left[\left(\partial_{\eta} \phi_{j}+\kappa\right) \sin \left(\phi_{i}+\theta\right)+c_{i}^{-1} \partial_{\tau} \phi_{i} \cos \theta\right] \sin \phi_{i}, \\
& \partial_{i} \sigma=a_{j} \partial_{\tau} \phi_{i} \sin \phi_{i}\left[1-\left(\sigma+\rho_{i}\right)^{-1} a_{i} \cos \phi_{i}\right] \text {, } \\
& \partial_{x_{1}} \eta=a_{i} c_{j}^{-1} \partial_{\tau} \phi_{i} \sin \left(\phi_{j}+\theta\right)+\left(\sigma+\rho_{i}\right)^{-1} a_{i}^{2} \\
& \cdot\left[\left(\partial_{\eta} \phi_{i}+\kappa\right) \cos \left(\phi_{i}+\theta\right)-c_{i}^{-1} \partial_{\tau} \phi_{i} \sin \theta\right], \\
& \cdot\left[\left(\partial_{\eta} \phi_{i}+\kappa\right) \sin \left(\phi_{i}+\theta\right)+c_{i}^{-1} \partial_{t} \phi_{i} \cos \theta\right], \\
& \partial_{t} \eta=-a_{j} \partial_{\tau} \phi_{i}\left[1-\left(\sigma+\rho_{i}\right)^{-1} a_{i} \cos \phi_{i}\right] \text {, }
\end{aligned}
$$

(A.10) $\partial_{t} \tau=a_{i}\left(\partial_{\eta} \phi_{i}+\kappa\right)+\left(\sigma+\rho_{i}\right)^{-1} a_{j}^{2} c_{i}^{-1} \partial_{\tau} \phi_{i} \sin \phi_{i} \cos \phi_{j} \cdot$

When $L_{i}$ is applied to a function independent of $t$, it reduces to $\Delta$. If $\phi_{i}$ is also independent of $\tau$ then

$$
L_{i}=\Delta=\sum_{i=1}^{2}\left[\left(\partial_{x_{i}} \sigma\right) \partial_{\sigma}+\left(\partial_{x_{i}} \eta\right) \partial_{\eta}\right]^{2}
$$

Upon using (A.4), (A.5), (A.7) and (A.8) in (A.11) and simplifying the result, we obtain

$$
\begin{aligned}
\Delta=(\sigma+ & \left.\rho_{i}\right)^{-2} a_{i}^{2}\left[\partial_{\eta}^{2}-2 \sin \phi_{j} \partial_{\eta} \partial_{\sigma}+\sin ^{2} \phi_{j} \partial_{\sigma}^{2}\right] \\
& +\partial_{\sigma}^{2}+\left[\left(\sigma+\rho_{i}\right)^{-2} a_{i} \partial_{\eta} a_{i}+a_{i}^{2}\left(\sin \phi_{i}-\partial_{\eta} \rho_{i}\right)\left(\sigma+\rho_{i}\right)^{-3}\right] \partial_{\eta} \\
& +\left[\left(\sigma+\rho_{i}\right)^{-1}-\left(\sigma+\rho_{i}\right)^{-2} a_{j}\left(\partial_{\eta} a_{i} \sin \phi_{i}+\partial_{\eta} \phi_{i} a_{i} \cos \phi_{i}\right)\right. \\
& \left.+\left(\sigma+\rho_{i}\right)^{-3} a_{j}^{2}\left(\partial_{\eta} \rho_{i}-\sin \phi_{j}\right) \sin \phi_{i}\right] \partial_{\sigma} .
\end{aligned}
$$




\section{ReFERENCES}

[1] F. C. Karal JR. \& J. B. Keller, Elastic wave propagation in homogeneous and inhomogeneous media, J. Acoust. Soc. Am., 31 (1959) 694-705.

[2] R. M. Lewrs, Proc. Symp. on Quasi-Optics, Polytechnic Press, Polytechnic Inst. of Bklyn., 1964, 71-103.

[3] D. S. Ahluwalia \& R. M. Lewis., J. Inst. Math. Applics., 5 (1969).

[4] J. B. Keller, R. M. Lewis \& B. D. Seckler, Asymptotic solutions of some diffraction problems, Comm. Pure Appl. Math., 9 (1956) 207-265.

[5] E. Resende, Propagation, Reflection and Diffraction of Elastic Waves, $\mathrm{Ph}$. D. Thesis, New York Univ., N. Y., 1963.

[6] R. Courant \& D. Hilbert, Methods of Mathematical Physics, Vol. 2, Interscience, New York, 1962.

[7] D. Ludwig, Exact and asymptotic solutions of the Cauchy problem, Comm. Pure Appl. Math., 13 (1960) 473-508.

[8] F. G. Friedlander, Simple progressive solutions of the wave equation, Proc. Camb. Phil. Soc., 43 (1947) 360-373.

[9] F. G. Friedlander, Sound Pulses, Cambridge Univ. Press, 1958.

[10] F. J. Gilbert, Scattering of impulsive elastic waves by a smooth convex cylinder, Acoust. Soc. Am., 32 (1960) 841-857.

[11] H. B. Keller, Propagation of stress discontinuities in inhomogeneous elastic media, SIAM Review, 6 (1964) 356-382.

[12] J. B. Keluer \& R. M. Lewis, Asymptotic Theory of Wave Propagation and Diffraction, Wiley, New York, to be published.

[13] D. Ludwig, Uniform asymptotic expansions at a caustic, Comm. Pure Appl. Math., 19 (1966) 215-250.

Courant Institute

and

Department of Mathematics

University Heights

New York University

Date communicated: December 30, 1968 